\title{
Leishmaniasis of the upper aerodigestive tract: A lesson for pathologists
}

\author{
Badr AbdullGaffar, Omar Nazhat, Rashid Mustafa, Omar Farouq \\ Pathology section, Rashid hospital, Dubai, United Arable Emirates \\ Correspondence: Badr AbdullGaffar, MD. Address: Pathology section, Rashid hospital, Dubai, UAE. \\ Email: badraah009@yahoo.com \\ Received: January 7, 2014 \\ Accepted: February 23, $2014 \quad$ Online Published: February 27, 2014 \\ DOI : $10.5430 /$ crcp.v1n1p17 \\ URL: http://dx.doi.org/10.5430/crcp.v1n1p17
}

\begin{abstract}
Primary mucosal leishmaniasis (ML) is relatively rare. The upper aerodigestive tract (UADT) is prone to ML in endemic areas and in immunocompromised patients. ML is gaining noticeable occurrence in non-endemic countries because of travel. Chronic secondary ML in non-endemic areas could be overlooked by the unwary clinicians and histologically missed by pathologists. This might result in erroneous treatment, acute exacerbation, disseminated disease and hindered recovery due to delayed diagnosis and therapy. We report a case of a 49-year-old man, who presented with dysphagia and hoarseness of the voice for one year. Clinically, leishmaniasis was unsuspected and scant amastigotes were histologically missed in two consecutive biopsies of the UADT. The patient was treated with corticosteroids for chronic inflammation and lymphoid hyperplasia, which resulted in worsening condition and a disseminated disease. The third biopsy showed numerous amastigotes. The diagnosis was flaring of ML with dissemination in a patient with chronic disease due to iatrogenic immunosuppression. He was treated with amphotericin B with incomplete recovery.
\end{abstract}

\section{Key words}

Leishmaniasis, Mucosal, Pharynx, Larynx

\section{I ntroduction}

Although mucosal involvement of the upper aerodigestive tract (UADT) in cutaneous or visceral leishmaniasis is frequent in the New World (Latin Americas), it is challenging for the clinicians and pathologists in the Old world (Europe, North Africa, Middle East and Asia) and in North America, because it is less frequent and clinically unsuspected due to lack of specific clinical and radiologic findings ${ }^{[1]}$. Chronic lesions might manifest years after initial infection and could be missed. This could result in delayed detection and late or wrong management which might lead to acute exacerbation, disseminated disease and hindered recovery ${ }^{[1-3]}$. Therefore, clinicians and pathologists should be aware of the fact that mucosal leishmaniasis (ML) of the UADT is not uncommon and might exist with or without cutaneous or visceral lesions.

\section{Case report}

A 49-year-old non-smoking, previously healthy, man presented with chronic progressive dysphagia and hoarseness of the voice for one year. He also complained of weight loss and fatigue. Blood tests showed raised erythrocyte sedimentation 
rate $(77 \mathrm{~mm} / 1 \mathrm{hr}), \mathrm{C}$-reactive protein $(66 \mathrm{mg} / \mathrm{L})$, low white blood cell count $\left(2.9 \times 10^{3} / \mu \mathrm{L}\right)$, low red blood cell count $\left(4.2 \times 10^{6} / \mu \mathrm{L}\right)$, low hemoglobin $(11.8 \mathrm{~g} / \mathrm{dL})$ and low platelets count $\left(133 \times 10^{3} \mu \mathrm{L}\right)$. Differential count showed relative neutrophilia (absolute neutrophils of $3.6 \times 103 / \mathrm{uL}$ and relative neutrophils of $88 \%$ ), and absolute lymphocytopenia (absolute lymphocytes of $0.2 \times 10^{3} \mu \mathrm{L}$ and relative lymphocytes of $4 \%$ ). Liver function tests showed high globulin $(6.9 \mathrm{~g} / \mathrm{dL})$, low albumin $(3.1 \mathrm{~g} / \mathrm{dL})$, raised alkaline phosphatase $(157 \mathrm{U} / \mathrm{L})$ and raised alanine aminotransferase $(48 \mathrm{U} / \mathrm{L})$. Serology tests for hepatitis viruses, human immunodeficiency virus (HIV), and T-spot test for tuberculosis were negative. Anti-nuclear factor was raised. Initially, the patient was admitted to the otorhinolaryngology unit and a panendoscopy and a CT-examination showed a diffuse involvement of the nasopharynx, oropharynx, hypopharynx and the larynx by an erythematous nodular to polypoid swollen lesion (see Figure 1). The lesion also involved the right maxillary sinus, the right mastoid air cells and the right middle ear. Enlarged right submandibular lymph nodes and bilateral upper cervical lymph nodes were also detected (see Figure 1). The clinical and radiologic differential diagnoses included chronic inflammation, infection, lymphoid hyperplasia and lymphoma. The first biopsy was taken from the nasopharynx and showed diffuse plasmahistiocytic inflammatory infiltrate, fibrosis and epithelial hyperplasia. The patient was treated with a course of antibiotics and mucolytics for 3 months without improvement.

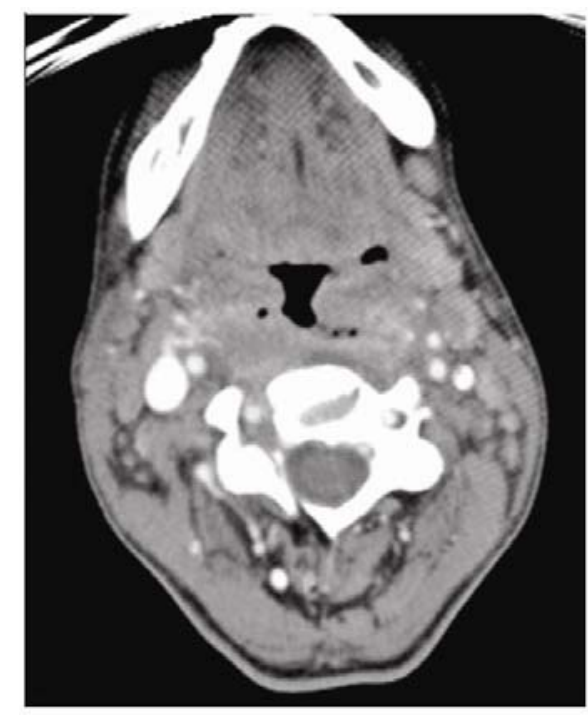

Figure 1A. A cross CT scan of the oropharyngeal area shows an irregular diffuse nodular thickening of the pharyngeal wall and narrowing of the lumen. Note the enlarged submandibular lymph nodes.

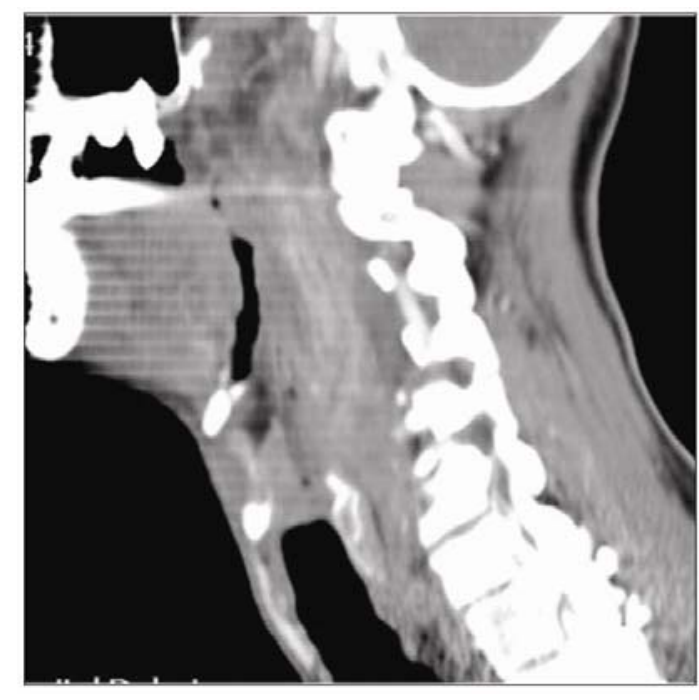

Figure 1B. A longitudinal CT scan of the head and neck region shows a diffuse thickening of the nasopharynx, oropharynx, hypopharynx and larynx with narrowing of the lumen. (Note: The clarity of the CT scan figures are suboptimal due to machine artifacts)

A second biopsy from the nasopharynx, oropharynx, hypopharynx and vocal cords was taken. It showed similar histomorphologic features to the previous biopsy and was interpreted as chronic, predominantly lymphohistiocytic, inflammation with no specific pathologic diagnosis. Suggested differential diagnoses included deep fungal infection, atypical mycobacterial infection, autoimmune diseases, and nonspecific inflammatory reaction at the periphery of a deeper neoplastic lesion. PAS and GMS stains for fungi and ZN stain for acid-fast bacilli were reported negative in biopsies. Cultures for mycobacteria and fungi were also negative. On the basis of the initial pathology reports, the negative microbiology tests and the raised anti-nuclear factor; the patient was treated with several cycles of corticosteroids (dexamethasone $4 \mathrm{mg}$ twice daily) and mucolytics for 6 months. Reassessment showed worsening of the symptoms with extensive involvement of the UADT. The patient developed fever and tender abdomen. Abdominal ultrasound showed hepatosplenomegaly, which explains the pancytopenia due to splenic sequestration. A third biopsy was taken from the oropharynx and cricopharynx. It showed diffuse sheets of histiocytes with numerous intracellular leishmania amastigotes (see Figure 2) that were positive for Giemsa stain (see Figure 2), but negative for PAS, GMS and Mucicarmine stains. Review of the two previous biopsies showed few similar parasites (see Figure 2) that were missed by the original 
pathologists. The parasites were round to oval basophilic structures with eccentric kinetoplast. Occasionally, the organisms were arranged at the periphery of the macrophages in a ring fashion. They were negative for PAS, GMS and Mucicarmine, positive for Giemsa, and lacked a capsule, which help distinguish them from look-a-like organisms, for example Histoplasma and Cryptococcus. Serology tests for leishmania antibody showed a raised titer (1:256). The patient gave a history of travel to Somalia, but he did not recall a history of insect bite and no cutaneous lesions were identified. The patient was diagnosed as a case of mucosal leishmaniasis (ML) of the UADT with flaring of the chronic lesions and disseminated disease due to prolonged iatrogenic immunosuppression. He was treated with liposomal amphotericin B (3 $\mathrm{mg} / \mathrm{kg} / \mathrm{per}$ day) for 7 days, then once a week for 5 weeks. A follow up of three months showed mild improvement of his signs and symptoms.

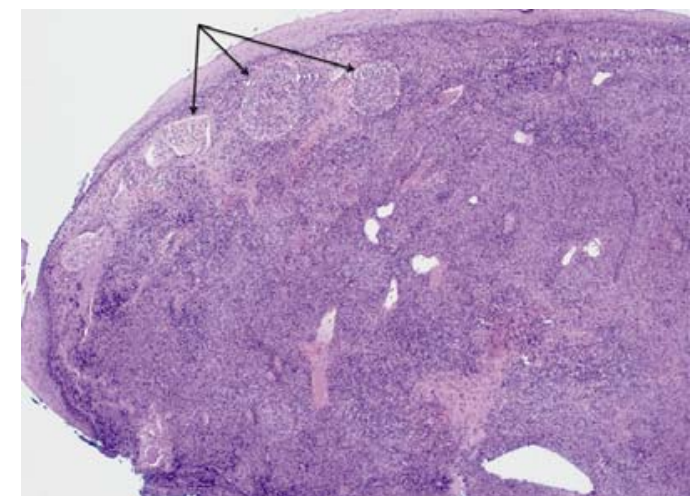

Figure 2A. The third biopsy shows diffuse solid sheets of inflammatory infiltrate replacing the stroma with an overlying focally ulcerated surface squamous epithelium. Note the dilated lymphatic vessels (arrows) (hematoxlyin-eosin, original magnifycation $\times 40$ ).

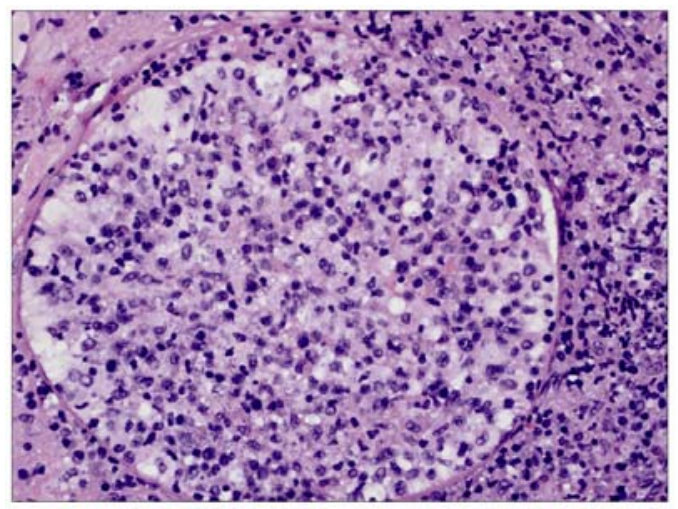

Figure 2C. A dilated lymph vessel filled with parasitized histiocytes. The endothelium is focally disrupted and infiltrated by histiocytes (hematoxlyin-eosin, original magnification $\times 400$ ).

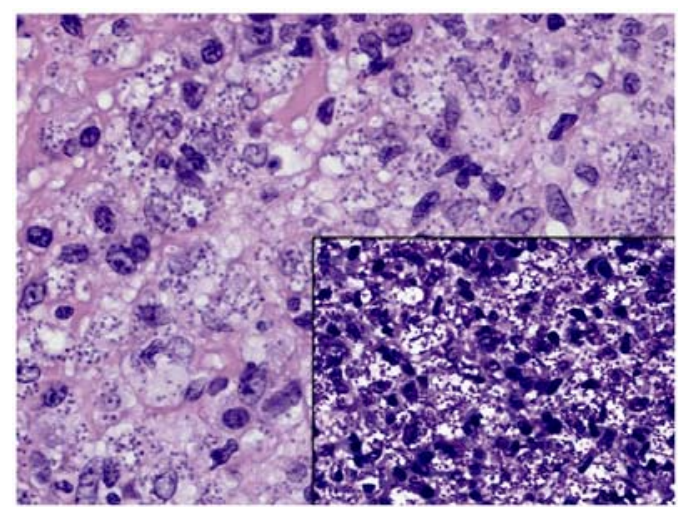

Figure 2B. A prominent histiocytic infiltrate with numerous intracellular parasites, and few plasma cells in the background. (hematoxlyin-eosin, original magnification $\times 1000$ ). Inset shows numerous intracellular amastigotes staining dark purple to blue with Giemsa stain (Giemsa, original magnification $\times 1000$ ).

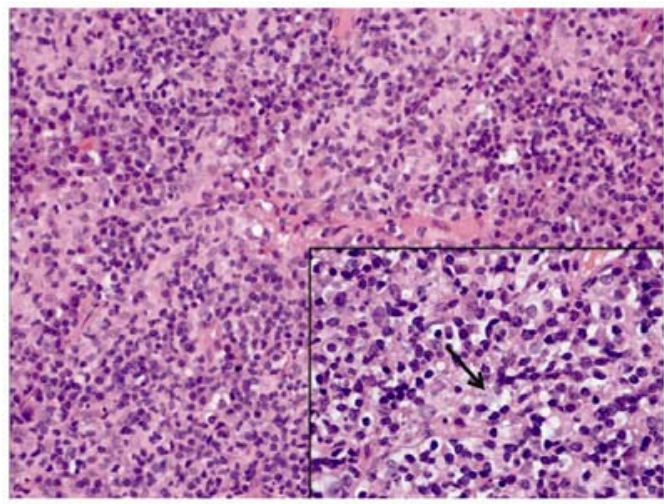

Figure 2D. The first two biopsies showed similar histomorphology of diffuse chronic inflammatory infiltrate predominantly composed of plasma cells and few histiocytes. Arrow points to the scant intracellular parasites that were initially missed (hematoxlyin-eosin, original magnification $\times 1000$ ).

\section{Discussion}

ML is gaining more prevalence in European and rapidly developing countries because of travel to endemic zones ${ }^{[1-5]}$. Higher rates of ML are noticed in patients with acquired immunodeficiency syndrome (AIDS) and immunosuppressed patients ${ }^{[1-3,5]}$. Even though L. brazilinesis is the main culprit of ML in the New World, nowadays other species, for 
example, L. infantum, and L. donovani can primarily present as oral, nasal, pharyngeal and laryngeal mucosal lesions in non-endemic countries ${ }^{[1-5]}$. The parasites spread to mucosal areas via lymphatic or hematogenous routes. The mechanism of tropism of L species to the UADT is related to parasitic and host factors ${ }^{[4]}$. Poor oral hygiene and manipulated host innate defenses of the UADT due to smoking, air pollution, allergy or chronic inflammation provide a suitable milieu for the parasite ${ }^{[4,5]}$. Tropism of certain $\mathrm{L}$ species is probably linked to the unique environment of the UADT ${ }^{[1,5]}$. Some consider the lower temperature and variable $\mathrm{pH}$ of the UADT might help the survival of certain adapted L strains by shedding certain enzymes ${ }^{[1,5]}$. The closely linked lymphatic drainage and reticuloendothelial system of the UADT help rapidly spread the parasites within mucosal and submucosal tissues and into the draining lymph nodes ${ }^{[2]}$. Finally, immunosuppression predisposes to $\mathrm{ML}^{[2]}$. Patients infected with HIV or medically immunosuppressed tend to have higher rates of $\mathrm{ML}^{[2]}$.

Our case highlights the fact that ML can be missed and might be confused with other UADT infectious, non-infectious and neoplastic lesions ${ }^{[1]}$. Iatrogenic immunosuppression might convert chronic lesions with a low number of parasites into acute lesions with numerous parasites resulting in severe inflammatory reaction causing extensive local swelling with worsening symptoms, extensive damage to pharyngeal tissues and access to lymphatics and blood vessels resulting in a disseminated disease. A meticulous search for the parasites in chronic lesions of the UADT with a history of travel to endemic areas should be advocated by pathologists. The histologic appearance of chronic lesions can be non-specific. Differential diagnoses might include tuberculosis, histoplasmosis, crytpococcosis and noninfectious inflammatory reactive processes. Tissue histology with Giemsa stain is the most common established way to spot the amastigotes ${ }^{[1,2]}$. Although it is highly specific, its sensitivity is variable depending on the abundance of the organisms which depends on the type of $L$ species and the stage of disease ${ }^{[2]}$. Leishmania parasites should be distinguished from mimickers. Histoplasma organisms are basophilic dots with a surrounding artifactual clear halo. They stain with PAS and GMS. Cryptococcus organisms are ovoid or spheric occasionally budding structures. They have a refractile wall that stains with PAS and GMS, and a capsule that stains with Mucicarmine. They lack the small rod-like kinetoplast characteristic of L species and do not stain with Giemsa. In equivocal cases, immunohistochemical and isoenzyme profiling and blood antileishmania antibodies confirm the diagnosis, albeit being time consuming ${ }^{[1,2,5]}$. Nowadays polymerase chain reaction (PCR) is a highly sensitive, specific and a faster confirmatory test, but it is not always available ${ }^{[2,5]}$.

Some might argue that other similar case reports and case series were reported in the literature and that our case does not add new information. However, cases of ML need to be reinforced periodically because they might represent under reported aspects of this lesion in non-endemic countries. It also demonstrates the fact that the absence of cutaneous lesions or lack of history of insect bites should not preclude clinicians from suspecting chronic ML of the UADT even in patients with a long history of travel. We agree that it is not a very rare or an extraordinary situation, but it has to be considered by clinicians and pathologists in the differential diagnoses of similar conditions. Finally, our case has important therapeutic implications in that erroneous iatrogenic immunosuppression could transform a localized chronic disease into a life-threatening obstructive, locally destructive, disseminated and therefore difficult to treat disease.

Treatment of leishmaniasis is mainly by pentavalent antimonials and liposomal amphotericin $\mathrm{B}^{[1-5]}$. Proper management of ML of the UADT is controversial because of the relatively limited number of reported cases, limited experience, risk of treatment complications and risk of late recurrence ${ }^{[1-3,5]}$. In our case, making the correct diagnosis earlier would have avoided the unnecessary immunosuppression and prompted earlier adequate treatment. The delayed therapy, prolonged immunosuppression that resulted in acute exacerbation and wide spread of the lesion, and malnourishment due to worsening dysphagia have caused partial recovery of the patient.

In conclusion, ML of the UADT is not uncommon presentation in endemic areas. However, it is less frequent in non-endemic countries and can be diagnostically challenging for the clinicians and pathologists. A raised level of clinical suspicion should help pathologists search carefully for the protozoan in biopsies. This will avoid unnecessary delay in diagnosis, prevent harmful erroneous immunosuppression and prompt early therapy with a better recovery. 


\section{References}

[1] Aliaga L, Cobo F, Mediavilla JD, et al. Localized mucosal leishmaniasis due to Leishmaniainfantum: clinical and microbiologic findings in 31 patients. Medicine (Baltimore). 2003; 82: 147-158. http://dx.doi.org/10.1097/01.md.0000076009.64510.b8

[2] Strazzulla A, Cocuzza S, Pinzone MR, et al. Mucosal Leishmaniasis: An underestimated presentation of a neglected disease. BioMed Res Intern. 2013; 2013:805108. http://dx.doi.org/10.1155/2013/805108

[3] Amato VS, Tuon FF, Imamura R, et al. Mucosal leishmaniasis: description of case management approaches and analysis of risk factors for treatment failure in a cohort of 140 patients. J EurAcadDermatolVenereol. 2009; 23: 1026-1034. http://dx.doi.org/10.1111/j.1468-3083.2009.03238.x

[4] Lessa MM, Lessa HA, Castro TW, et al. Mucosal leishmaniasis: epidemiological and clinical aspects. Braz J Otorhinolaryngol. 2007; 73: 843-847.

[5] Cocuzza S, Strazzulla A, Pinzone MR, et al. Isolated laryngeal leishmaniasis in immunocompetent patients: An underdiagnosed disease. Case Rep Infect Dis. 2013: 165409. http://dx.doi.org/10.1155/2013/165409 